\title{
A Design of Antenna with Pattern Reconfigurable Characteristic Working on Ka band
}

\author{
Deng Zhong Liang \\ School of Electronic Engineering \\ Beijing University of Posts and Telecommunications \\ Beijing, P.R. China \\ dengzhl@bupt.edu.cn
}

\begin{abstract}
One pattern of patch antenna with the function of reconfiguration is presented. This antenna is composed of one rectangle patch and two $U$-shape patches.They can be connected by RF MEMS (micro electromechanical systems for RF circuits) switches. By controlling the states of these RF MEMS switches, the antenna can radiate four patterns. The four patterns have the same operating frequency at $35 \mathrm{GHz}$. Ansoft HFSS provides the simulation results. This antenna can be used in radar, satellite, security communications and so on.
\end{abstract}

Keywords- pattern reconfigurable; ka band; RF-MEMS; Microstrip patch antenna

\section{INTRODUCTION}

In morden communication systems, reconfigurable antennas show many great superiorities because they can afford diversity features in operating frequency, polarization and radiation pattern. The operation of an antenna's radiation pattern can be used for detecting target signals and avoiding electronic interference or noise. In many cases, these antennas can enhance the security of telecommunication. For all above reasons, there is a great demand for radars, wireless communications, satellite communications fields and so on [18]. The pattern reconfiguration feature is usually attained by phase-shifted antenna arrays, but the size, weight and complexity of installation may not satisfy the requirement of many applications. Recently, more and more designs are proposed to reconfigure the antenna, in which switching mechanisms are extensively used to change the current paths, control the current distribution and provide pattern reconfigurable behavior[2-4]. Microstrip patch antenna plays great role in the reconfigurable antenna field due to its advantages such as low profile, light weight and small size, and compatible with MMIC design.

RF MEMS switches[5-7] have the advantage of low insert loss and high isolation, so they attract many attentions for application in wireless communication systems.

In this paper, one novel reconfigurable micro strip antenna was proposed. By controlling RF MEMS switches to change the states of antenna, metal strips and air gaps are used rather than "off \& on" switches to simulate the states by the Ansoft HFSS software.

\author{
Wang Hui Jun \\ School of Electronic Engineering \\ Beijing University of Posts and Telecommunications \\ Beijing, P.R. China \\ huijun520@bupt.edu.cn
}

\section{ANTENNA STRUCTURE.}

The structure of the reconfigurable pattern micro strip is presented in Fig.1,. It is composed of by three part, center rectangle patch, two U-type patches and four switches which combine them. The center patch with its length and width fixed to be $1.15 \mathrm{~mm}$ and $0.65 \mathrm{~mm}$. The U-type patches' $\mathrm{s}$ dimension is $1.58 \times 3.15 \mathrm{~mm}^{2}$ and its removed part inside is a $0.95 \times 1.49$ $\mathrm{mm}^{2}$ rectangle. The other is the same to it. All the structure is placed on the same face of a Duroid 5880 substrate with $\varepsilon r=2.2$ and $\mathrm{h}=1.5 \mathrm{~mm}$. The ground plane has the same dimensions with the substrate fixed to be $4 \times 5 \mathrm{~mm}^{2}$.A probe feed of radius $0.09 \mathrm{~mm}$ is directly combined to the center of the center patch. Four switches are the same completely. The size of each switch is $0.3 \times 0.42 \mathrm{~mm}^{2}$.

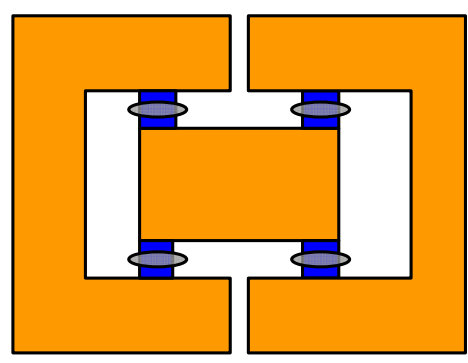

Fig.1. The structure of the Antenna

The four switches of the antenna can be put separately expressed four kind of states as Fig .2, which simulate the four states Intuitively
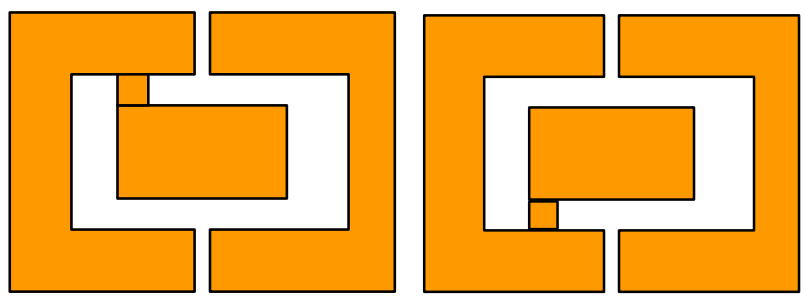

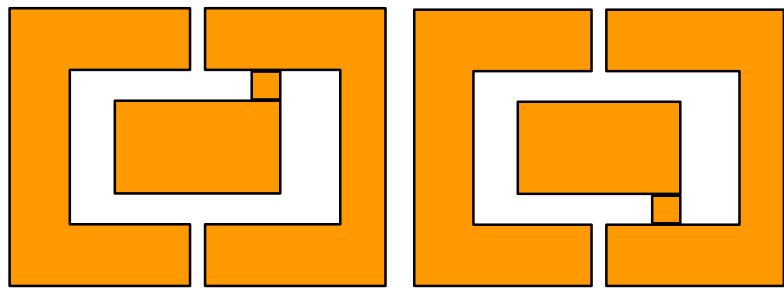

Fig.2. The four simulated statess in the ideal conditions

\section{SimUlated ResUlts AND ANALYSIS.}

In order to get the performance of this antenna, with the Ansoft HFSS electromagnetic simulation, the performance of the proposed antenna was simulated. Comparing to using silicon( $(\varepsilon r=11.9)$, the electromagnetic field of the meterial we chose are more loosely bound and it can radiate into the open space more easily. The S parameter S11 for the proposed microstrip patch antenna at one state with single switch closed is shown in Fig.3

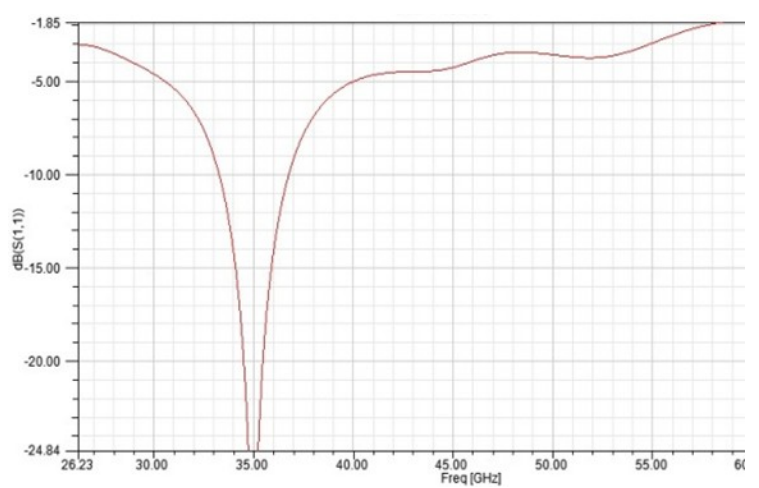

Fig.3. The simulated S parameters S11 for the RF-MEMS switch at four states

Fig.4. reveals the VSWR(Voltage Standing Wave Ratio) simulation results, it indicates that the VSWR is lower than 2.0 at the working frequency $35 \mathrm{GHz}$.

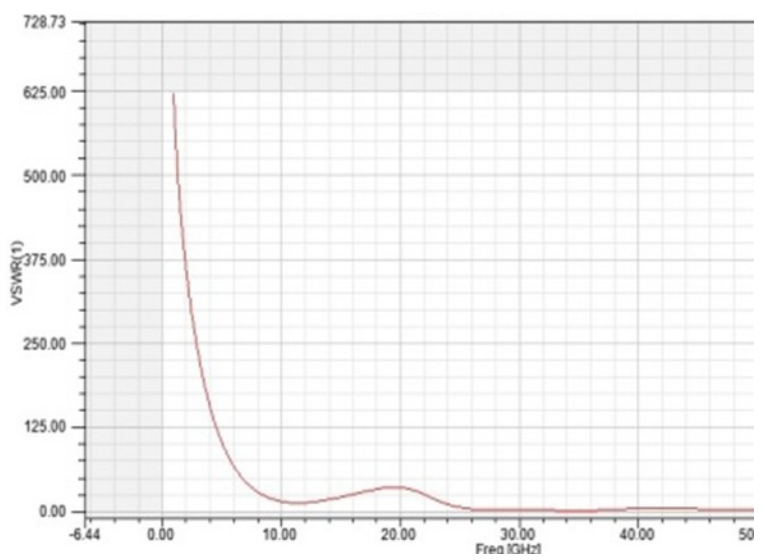

Fig.4. The simulated VSWR for the RF-MEMS switch at four states

The radiation patterns of four states in the $\mathrm{E}$ plane( $\mathrm{x}-\mathrm{Z}$ plane)Fig. 5 and $\mathrm{H}$ plane(y-z plane)Fig. 6 are presented .In Fig.5 or Fig.6, one switch is used.In the case,a relative impedance bandwidth of $10 \%$ was obtained around the resonant frequency $35 \mathrm{GHz}$,over which the return losses were better than $30 \mathrm{~dB}$.
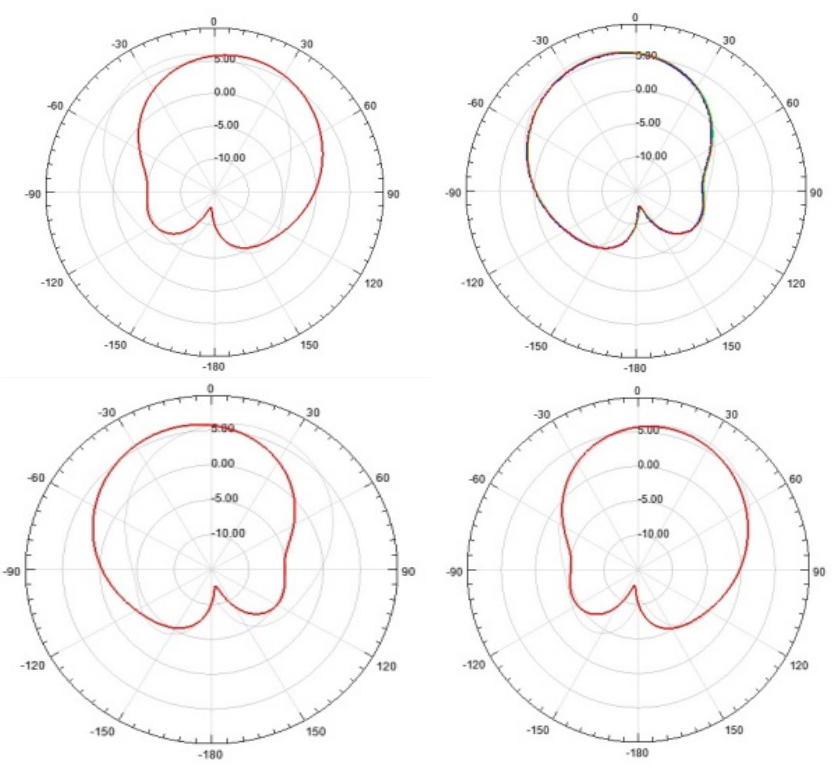

Fig.5. The radiation pattern in E-plane
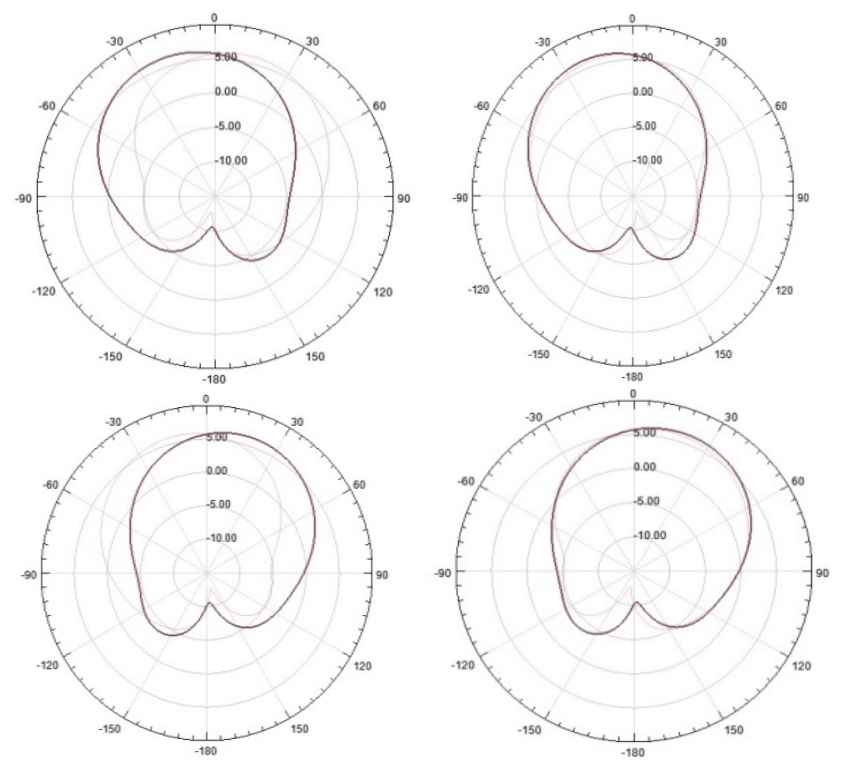

Fig.6. The radiation pattern in H-plane

The Fig. 7 presents the 3D simulation effects of four states, it more intuitive show that this design can radiation to space four different directions.

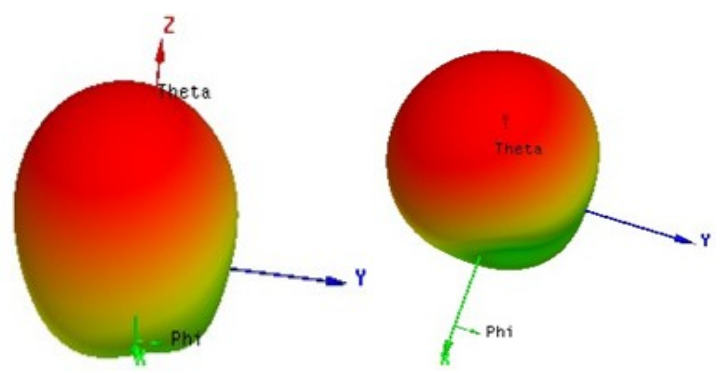




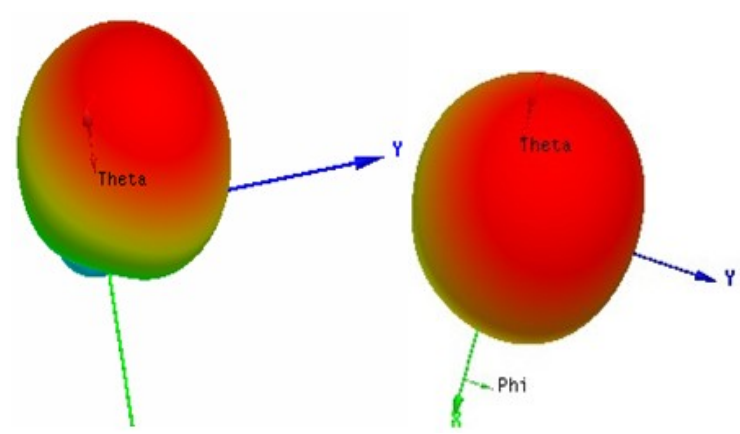

Fig.7. The 3D radiation patterns

\section{RF-MEMS SWITCHES}

Fig. 8 shows the structure of the RF MEMS switch used for the aforesaid reconfigurable microstrip patch antenna. The RFMEMS switch consists of a lower electrode fabricated on the surface of the IC and a thin aluminum membrane suspended over the electrode. The membrane "bridge" suspended over the center conductor of a coplanar waveguide (CPW) or microstrip line and fixed at both ends to the ground conductors of the $\mathrm{CPW}$ line. The bridge can be actuated from an up state to a down state. In the up state, the bridge is relaxed and the air gap is presented between the dielectric and the bridge. In the down state, an electrostatic force is applied by an external control voltage to collapse and eliminate the air gap between the dielectric and the bridge.The characteristics of the dielectric layer primarily determine the MEMS on-capacitance.By virtue of changing the position of the membrane with an applied DC voltage, the capacitance of this device can be changed over a significant capacitance range.

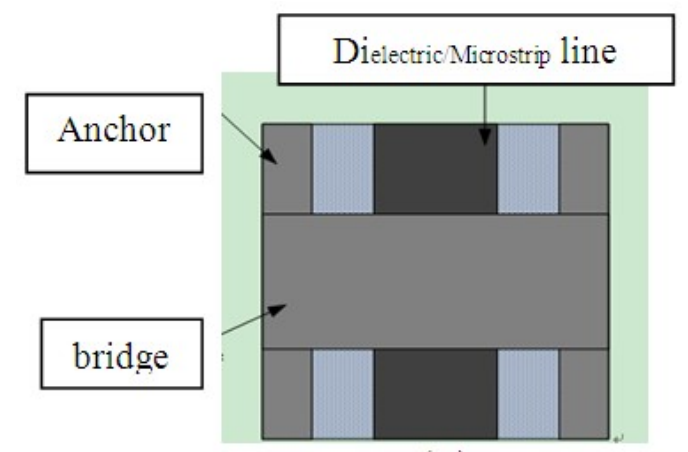

(a)

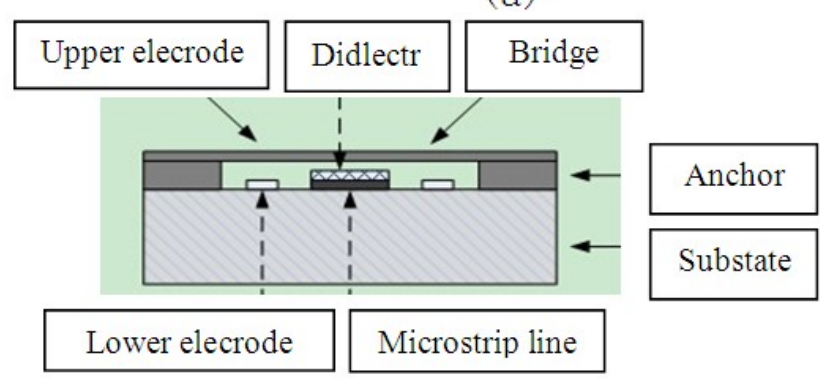

(b)
The simulated S parameters S11 and S21 for the RF MEMS switch at two states are shown in Fig.9.As is shown in Fig.9(a) at Up state ,the reflection coefficient is lower than $-20 \mathrm{~dB}$ and the transmission coefficient is larger than $-0.1 \mathrm{~dB}$, respectively over the Ka-band. So all energy could pass through via the switch. And at Down state, the reflection coefficient is larger than $-0.1 \mathrm{~dB}$, and the transmission coefficient is lower than $20 \mathrm{~dB}$, respectively over the Ka band, as is shown in Fig.9(b), the switch can be used to isolate the center patch from other two ones.

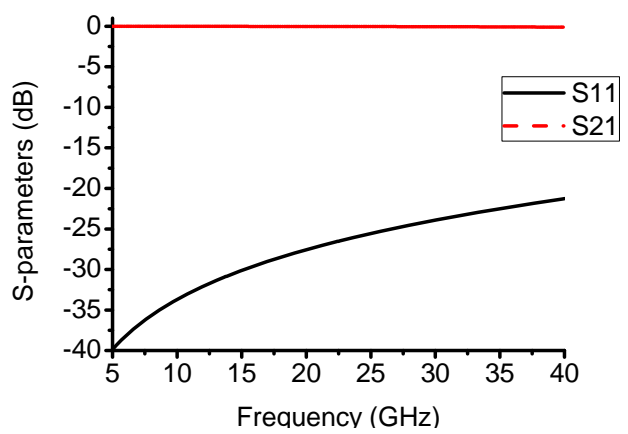

(a)

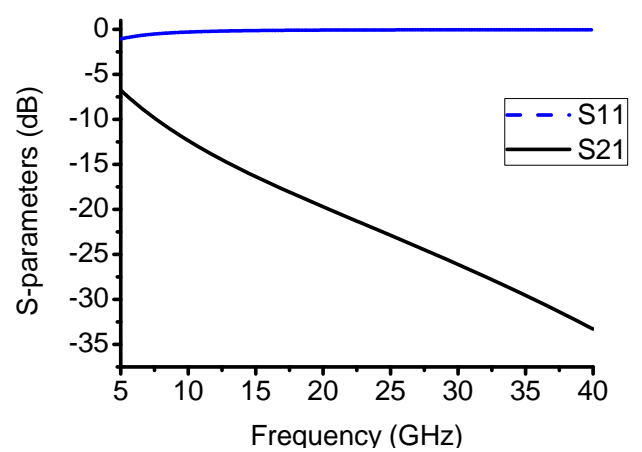

(b)

Fig.9. Diagrams of MEMS switch: (a) Up-State (b)Down-State

\section{CONCLUSIONS}

This is A ka-band microstrip patch antenna with switchable pattern. The antenna structure has four RF MEMS switches which have been used to control the states of connection thus changing the effective radiation structure of the antenna. The current distribution on the whole patch could be switched between four states. The antenna' structure has been simulated by Ansoft HFSS. From the results, it is found that the antenna can redirect four directions at its work frequency $35 \mathrm{GHz}$, With the covering rangers from $-15^{\circ}$ to $15^{\circ}$ in the E-plane, and from $-25^{\circ}$ to $25^{\circ}$ in the H-plane. The simulation results demonstrates that the design of the antenna can realize four wide scan range of orientations, These characteristics make the patch fancy for Ka-band communication. In the future work will highlight the integration with the four switches.

Fig.8. Diagrams of MEMS switch : (a)top view,(b)sectional view 


\section{ACKNOWLEDGMENT}

This work was financially supported by the Ministry of Education Funds, School of Electronic Engineering, Beijing University of Posts and Telecommunication, Beijing University of Posts and Telecommunication .Thanks

\section{REFERENCES}

[1] S.L.S Yang and K.M.Luk: IEEE Trans. Antennas Propag. VOL.54(2006),p.432-439

[2] S.H.Chen,J.S.Row and K.L. Wong:Reconfigurable Square-Ring Patch Antenna with Pattern Diversity ,IEEE Transactions on Antennas and Propagation, Vol.55 Issue 2(2007) p. 472-476
[3] J.B.Muldavin and G.M.Rebeiz: All-metal High-isolation Series and Series/Shunt MEMS Switch,IEEE Microwave and Wireless Components Letters, Vol.11 Issue 9(2001)p.370-377

[4] W.L.Liu,T.R.Chen,S.H.Chen and J.S.Row:Electron.Lett.Vol.43(2007)

[5] M/A-COM, RF and Microwave ICs and Discrete Semiconductors catalog,www.alphaind.com

[6] D.M.Pozar, Microwave Engineering, Addison-Wesley, Reading, MA, 1990.

[7] C. Buccella, M. Feliziani and G.Manzi: IEEE ISIE.VOl.3(2005),p.11171122

[8] C.W.Jung, M.J.Lee,G.P.Li and F.De Flaviis: Reconfigurable Scan-beam Single-arm Spiral Antenna Integrated with RF-MEMS Swithces, IEEE Transactions on Antennas and Propagation,Vol.54 Issue 2(2006)p.455465 